\title{
Technè
}

La science au service de l'histoire de l'art et de la préservation des biens culturels

$44 \mid 2016$

Archives de l'humanité : les restes humains patrimonialisés

\section{Les restes humains : questions de médiation}

L'exemple de l'école de médecine navale à Rochefort

Human remains : questions of mediation. The example of the School of Naval

Medicine, Rochefort

\section{Denis Roland}

\section{OpenEdition}

\section{Journals}

Édition électronique

URL : http://journals.openedition.org/techne/1001

DOI : 10.4000/techne.1001

ISSN : 2534-5168

Éditeur

C2RMF

Édition imprimée

Date de publication : 1 novembre 2016

Pagination : 45-47

ISBN : 978-2-7118-6339-6

ISSN : $1254-7867$

Référence électronique

Denis Roland, «Les restes humains : questions de médiation », Technè [En ligne], 44 | 2016, mis en ligne le 19 décembre 2019, consulté le 27 juillet 2020. URL : http://journals.openedition.org/techne/ 1001 ; DOI : https://doi.org/10.4000/techne.1001

La revue Technè. La science au service de l'histoire de l'art et de la préservation des biens culturels est mise à disposition selon les termes de la Licence Creative Commons Attribution - Pas d'Utilisation Commerciale - Pas de Modification 4.0 International. 


\section{Denis Roland \\ Les restes humains : questions de médiation. L'exemple de l'école de médecine navale à Rochefort}

Human remains: questions of mediation. The example of the School of Naval Medicine, Rochefort
Résumé. Les restes humains conservés dans les fonds patrimoniaux posent des questions de sensibilité et d'éthique qui tendent à occulter leur importance historique au profit d'une idée de musée des horreurs. Ce sentiment aboutit au mieux à une incompréhension, au pire à un rejet. Seule une médiation importante, soucieuse de comprendre et de faire comprendre, peut prétendre dépasser ces difficultés. L'école de médecine navale à Rochefort, gérée depuis trente ans par le musée national de la Marine, propose une expérience dans ce domaine encore peu explorée dans notre pays.

Mots-clés. Médiation, restes humains, Rochefort.

\begin{abstract}
Human remains held in cultural heritage collections raise questions of sensibility and ethics that tend to eclipse their historical import and promote the notion of a museum of horrors. At best, this leads to incomprehension; at worst, to rejection. Only informed mediation, striving towards understanding and making others understand, can overcome these difficulties. The School of Naval Medicine, Rochefort, run for the past three decades by the Musée National de la Marine, offers a positive experience in this domain, which, in France, is still virtually unexplored.
\end{abstract}

Keywords. Cultural project mediation, human remains, Rochefort.
La France conserve de très importantes collections d'histoire de la médecine, témoins de l'importance de la recherche et de la formation dans ce domaine depuis le Moyen Âge. À Montpellier, à Paris ou à Lyon, l'effort continu pour transmettre le savoir a conduit à constituer des ensembles riches et complets susceptibles de présenter le corps humain et ses pathologies dans leur plus grande diversité. Malgré de nombreuses destructions dues à la fragilité même des tissus organiques et au désintérêt progressif des universités de médecine envers ces outils d'enseignement, les restes humains forment aujourd'hui un patrimoine important mais problématique. Dépouillés de leurs fonctions éducatives, ils peinent à acquérir une valeur historique, pourtant incontestable, mais qui se heurte à des questions de sensibilité collective et d'éthique pas toujours bien posées. Conserver des cadavres est une chose, les exposer en est une autre et la question de la médiation autour des restes humains en est encore à ses débuts. Elle est pourtant essentielle pour éviter la mise en réserve et l'occultation d'une page d'histoire des sciences jugée trop choquante pour être lue. L'école de médecine navale à Rochefort poursuit de ce point de vue une expérience originale.

\section{Brève histoire d'un musée des horreurs}

$\mathrm{Au} \mathrm{XVII}^{\mathrm{e}}$ siècle, les chirurgiens-barbiers qui embarquent à bord des vaisseaux se signalent par leur dénuement et bien souvent leur franche incompétence, et la Marine de guerre qui se met alors en place a un besoin criant de professionnels de santé capables de faire face au désastre sanitaire d'un navire en mer. En 1722, dans l'arsenal du Ponant qu'est Rochefort depuis 1666, est fondée une école de chirurgie navale : c'est la première fois qu'un État prend ainsi en main la formation de ceux à qui elle confie la santé de ses équipages. Une pédagogie originale est mise au point, visant à former des praticiens efficaces, laissant les théories explicatives aux médecins de la faculté. Cette culture de l'observation marque durablement ces chirurgiens embarqués. Pragmatiques, ils étendent volontiers leurs pratiques très au-delà du seul domaine médical et s'emparent progressivement de la botanique, de la zoologie, de la géologie, de l'ethnologie. Les voyages d'exploration du XVIII ${ }^{\mathrm{e}}$ et, plus encore, du XIX ${ }^{\mathrm{e}}$ siècle les placent en position privilégiée pour participer à un ambitieux inventaire du monde qui les conduit à pousser assez loin un programme de rêve encyclopédique, fait de science transversale aux disciplines décloisonnées. De cette ambition, ils font un lieu. En 1788, un nouvel hôpital de la Marine est inauguré à Rochefort et l'un des pavillons est attribué à l'école de médecine ${ }^{1}$. Au cours du XIX ${ }^{\mathrm{e}}$ siècle, le lieu s'organise rationnellement sur trois niveaux : un rez-de-chaussée pour les 


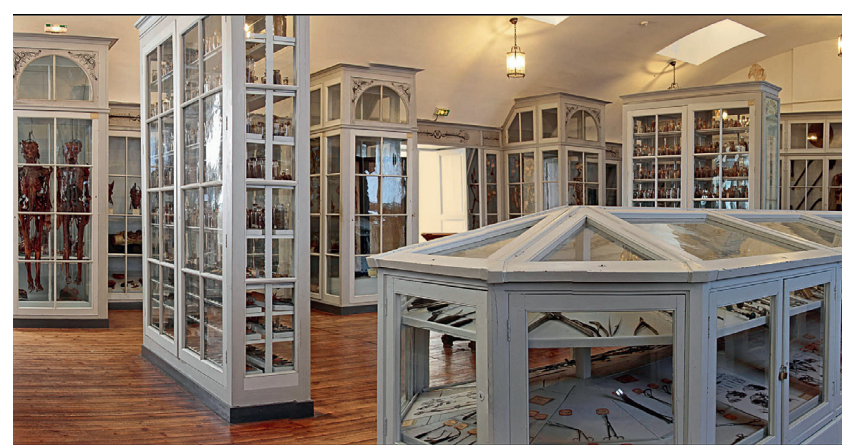

Fig. 1. Vue générale du musée de l'école de médecine navale. Les collections d'anatomie humaine y sont intégrées dans une présentation de 1862, où médecine et histoire naturelle se répondent, dans un idéal de monde classifié. (C) Musée national de la Marine/ J.-P. Brault.

espaces de prestige où l'institution se célèbre elle-même ; une vaste bibliothèque de 25000 volumes ; un musée présentant des échantillons et des objets en ce temps où la pédagogie ne peut être seulement livresque. En 1890, la création de l'École de Santé navale à Bordeaux fige la situation de l'établissement de Rochefort, qui devient école annexe. Jusqu'en 1963, des étudiants viennent y préparer le concours de Bordeaux, mais les collections n'interviennent plus dans le cursus, et l'espace est comme fossilisé dans son état de la fin du XIX ${ }^{\mathrm{e}}$ siècle. En 1986, le musée national de la Marine est sollicité pour rénover l'école et l'ouvrir au public. Les collections, très empoussiérées, sont restaurées et le lieu réhabilité dans une idée de restitution de l'état de 1890. Il ouvre au public en 1998. Témoin du rôle de la Marine dans l'histoire des sciences, au sein d'un arsenal patrimonial parmi les plus riches d'Europe, l'école de médecine conserve, après dix-huit ans de valorisation, une réputation tenace de lieu d'effroi et de dégoût, qui semble être le lot des toutes les collections d'histoire de la médecine.

\section{L'affect et la raison}

L'école de médecine navale est marquée par deux histoires : celle de la médecine navale et celle de l'histoire naturelle. Ses collections comptent plus de 7000 échantillons, spécimens ou objets, parmi lesquels les restes humains représentent 300 items, ainsi répartis : 40 éléments d'anatomie parmi lesquels deux écorchés, 130 pièces d'ostéologie, 80 spécimens d'anatomie pathologique et de tératologie et 50 crânes phrénologiques et raciaux. Même en y ajoutant une cinquantaine de pièces en cire et en carton, cet ensemble est quantitativement assez modeste, mais son impact sur l'ensemble du lieu est considérable.

La médiation mise en place par le musée depuis 1998 doit relever le défi singulier de rendre compréhensible à tous un lieu conçu pour des étudiants en médecine. La sensibilité collective de notre société dans laquelle l'on ne voit plus guère de cadavres est une difficulté majeure. L'imaginaire que dégage les collections d'anatomie humaine est extrêmement fort ; nous les lisons aujourd'hui avec des filtres qui vont des camps de concentration aux films d'horreur et peuvent conduire au malaise. Le bagage affectif et intellectuel des visiteurs est ici un redoutable inconnu dont il faut particulièrement tenir compte.

Les questions d'éthique, qui se refusent à réduire les restes humains au statut d'objets, est un autre volet du défi de la médiation. Les corps exposés proviennent d'individus qui, pour être anonymes, ne s'abordent pas moins avec la mémoire de ce qu'ils furent. En 2009 et 2010, l'interdiction à Paris de l'exposition Our body, à corps ouvert de Gunther von Hagens ${ }^{2}$ et l'affaire des têtes maories ${ }^{3}$ ont rappelé, s'il était besoin, toute l'actualité d'un débat très vif au sein de la société française. La tentation est grande de retirer de l'exposition les pièces les plus choquantes, comme les fotus conservés en bocal qui semblent être l'emblème même du musée des horreurs.

C'est un choix tout différent qui a été fait à Rochefort. Depuis 2003, toutes les visites sont accompagnées. L'objectif est de permettre aux visiteurs de ressentir pleinement la force affective du lieu, en accompagnant cette expérience d'un discours très rationnel. Ce discours est fréquemment revu avec l'équipe des guides. Contraignante pour les visiteurs comme pour le musée, cette médiation humaine est la seule qui permette l'adaptation au groupe et les échanges nécessaires à un débat qui doit rester ouvert. Elle offre aussi la possibilité de conserver en exposition la totalité des collections et de tordre le cou aux rumeurs qui entourent des collections mises en réserve, alimentant l'image du musée aux spécimens tellement épouvantables qu'on n'ose plus les montrer. Cacher, c'est donner libre court aux fantasmes les plus débridés ; montrer, c'est faire le pari de l'intelligence des visiteurs.

Fig. 2. Deux écorchés humains complets. Peu documentées, ces pièces anatomiques ont sans doute été réalisées à la fin du $\mathrm{XVIII}^{\mathrm{e}}$ siècle. Les individus dont le corps s'est ainsi trouvé préservé sont totalement anonymes. Le choix a été fait de conserver la présentation de la fin du $\mathrm{XIX}^{\mathrm{e}}$ siècle dont le musée de la Marine a hérité, de tenter de la comprendre et de la faire comprendre. (C) Musée national de la Marine/D. Boone. 


\section{De l'effroi à la culture}

Mais ce n'est pas tout. Transmettre sous forme de visites générales, longues ou courtes, un discours qui refuse le sensationnalisme n'est pas suffisant. Il faut aussi diversifier l'offre, ouvrir le lieu à toutes les formes d'expressions, dédramatiser un sujet complexe, mais que chacun doit percevoir comme un bien commun. En somme, il s'agit d'être un véritable acteur culturel sans rien renier de la nature des collections ni des difficultés qu'elles présentent. Depuis plus de dix ans, l'école de médecine navale a ainsi accueilli des spectacles, et souvent des créations, dans les domaines aussi divers que la musique (classique ou non), le théâtre, la danse, les marionnettes, le club littéraire ou les visites théâtralisées. Il n'est pas question de transformer l'école de médecine en salle de spectacle, mais d'explorer la richesse des thèmes et des atmosphères du lieu.

L'accueil des scolaires, singulièrement des élèves de primaire, ne va pas non plus de soi. Les discussions, parfois tendues, avec l'Éducation nationale n'ont jamais mis en question l'intérêt pédagogique du lieu, mais appréhendent les réactions de parents d'élèves susceptibles de mal comprendre que l'école puisse montrer des cadavres à leurs enfants. Sur cette question, qui n'a rien de simple, le musée de la Marine n'a pas seulement une opinion, mais une solide expérience : depuis dix ans, environ 10000 élèves ont été accueillis sur place, dont un peu plus de 2000 en primaire. Le résultat, qui vaut pour l'ensemble des enfants qui visitent le lieu, en famille ou avec la classe, montre combien le discours sur l'effort rationnel de générations de médecins pour comprendre le corps humain fonctionne avec des enfants, qui passent précisément leur temps, eux aussi, à comprendre le monde. Comme le disait un jour un enfant de 8 ans face à une pièce sèche de réseau artériel humain : On l'a en nous, mais on le voit jamais. On ne saurait mieux résumer. Profondément interdisciplinaires, les collections d'anatomie humaine ouvrent un champ très vaste d'exploitation pédagogique où se mêlent histoire, science et langage.
Sensibilité collective et éthique ne sont pas des obstacles à la valorisation des restes humains malgré l'affect très fort dont ils sont investis, entre peur, dégoût et voyeurisme. L'accompagnement des visiteurs et le respect nécessaire face aux collections doivent permettre d'intégrer pleinement l'histoire de la médecine dans le champ du patrimoine et de la culture commune. C'est un travail de longue haleine, mais la médiation sur les restes humains, dont les expériences sont encore trop peu nombreuses, est la condition première de leur conservation.

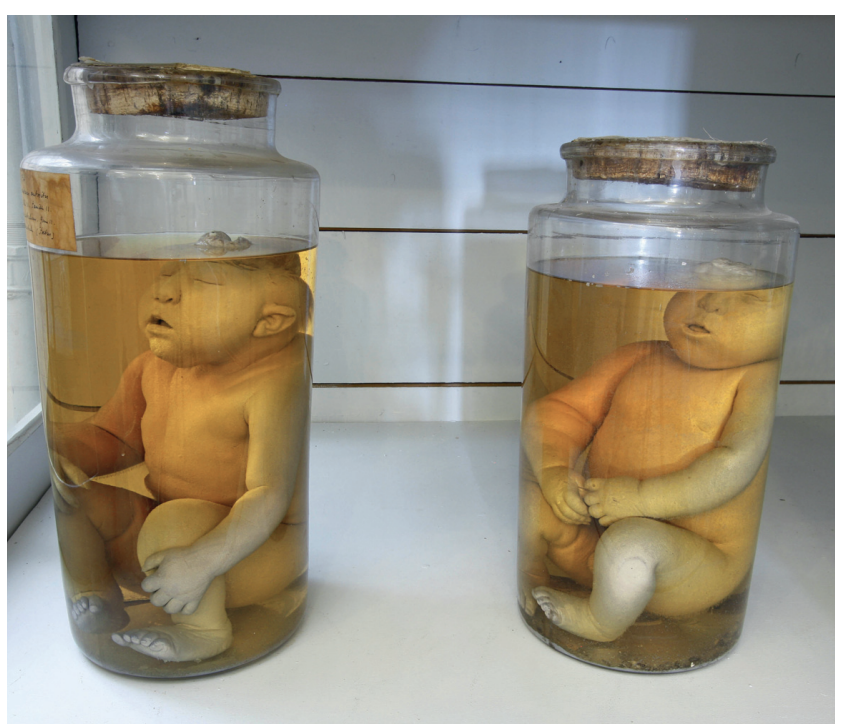

Fig. 3. Fœtus conservés en fluides. Ces deux fœtus font partie de la collection de tératologie en raison des malformations qu'ils présentent, et qui relèvent de la classification de Geoffroy Saint-Hilaire. Leur caractère impressionnant, voire choquant, fait facilement oublier le débat scientifique dont ils témoignent, celui de l'évolution des espèces. (๑) Musée national de la Marine/ M. Benhamou.
Notes

1. Morgat et Roland, 2016.

2. L'exposition, ouverte en février à l'Espace 12, présentant 17 cadavres conservés selon le procédé de la plastination, après avoir été présenté à Lyon et à Marseille, a été interdite en avril 2010 en raison de doutes sur la provenance des corps. La presse locale et nationale a également rendu compte des questions d'éthique posées par l'exposition d'écorchés. Voir par exemple Le Monde du 24 avril 2009.

3. Après un débat complexe, la France a fait droit à la demande de la NouvelleZélande et a restitué, en janvier 2012, 21 têtes momifiés de guerriers maoris faisant partie des collections publiques françaises. L'une de ces têtes faisait partie des collections de l'école de médecine navale de Rochefort.

\section{Bibliographie}

L'ancienne école de médecine navale, Paris, musée national de la Marine, 2006.

Cadot L., 2009, En chair et en os : le cadaure au musée. Valeurs, statuts et enjeux de la conservation des dépouilles patrimonialisées. Paris, École du Louvre/RMN.
Cadot L., 2007, «Les restes humains : une gageure pour les musées? ", La lettre de l'OCIM, $\mathrm{n}^{\circ} 109$, p. 4-15.

Morgat A. et Roland D., 2016, « L'hôpital de la Marine de Rochefort ", dans Revue de Société Française d'histoire des Hôpitaux, $\mathrm{n}^{\circ} 2016$.

«Restitution des restes humains. Tableronde au festival de cinéma RochefortPacifique », 2013, Journal de la Société des Océanistes, n¹36-137, p. 89-102. 